\title{
Which Neural Tract Plays a Major Role in Memory Impairment After Multiple Cerebral Infarcts? A Case Report
}

\author{
Dae Kwon Park, MD, Ki Hyun Byun, MD, Dongseok Yang, MD, PhD \\ Department of Physical Medicine and Rehabilitation, Ulsan University Hospital, \\ University of Ulsan College of Medicine, Ulsan, Korea
}

\begin{abstract}
Injury to the thalamocortical tract (one in the Papez circuit) that leads to memory impairment following brain injury is very rare. In this study, we present a case of partial injury to the thalamocortical tract that causes memory impairment after concurrent thalamic and hippocampal infarct. A 20-year-old male complained of memory impairment 1 month after partial injury to the thalamocortical tract. Using a probabilistic diffusing tensor tractography, it was found that the right thalamocortical tract was thinner than the left thalamocortical tract. However, all other neural tracts including the fornix, cingulum, and mammillothalamic tract were intact on both hemispheres. Therefore, the memory impairment in this patient was considered as being due to thalamic infarct based on the observation that the fornix from hippocampal infarct was intact. This case suggests that the assessment of lesions in the neural tracts of the Papez circuit might be useful for understanding the mechanism of memory impairment following cerebral infarction.
\end{abstract}

Keywords Cerebral infarction, Limbic system, Memory deficit

\section{INTRODUCTION}

Post-stroke memory impairment is the second-most common cause of dementia. Its prevalence is $11 \%-31 \%$ in non-demented patients 1 year after stroke [1]. Post-stroke impairment decreases the quality of life of a patient. It may even make it difficult for patients to return to normal life.
The limbic system is deeply responsible for the function of memory and behavior. The Papez circuit is composed of limbic gray and white matter. The circuit begins in the hippocampus, which is connected to the mammillary body via the fornix. The mammillary body is then connected to the anterior thalamic nuclei via the mammillothalamic tract (MTT). Next, the anterior thalamus is connected to the cingulum via the thalamocortical

Received August 2, 2017; Accepted October 12, 2017

Corresponding author: Dongseok Yang

Department of Physical Medicine and Rehabilitation, Ulsan University Hospital, University of Ulsan College of Medicine, 877 Bangeojinsunhwan-doro, Dong-gu, Ulsan 44033, Korea. Tel: +82-52-250-8940, Fax: +82-52-250-7211, E-mail: fnew1@hanmail.net

ORCID: Dae Kwon Park (https://orcid.org/0000-0002-4083-163X); Ki Hyun Byun (https://orcid.org/0000-0001-5506-5635); Dongseok Yang (https:// orcid.org/0000-0003-0355-866X).

(c) This is an open-access article distributed under the terms of the Creative Commons Attribution Non-Commercial License (http://creativecommons.org/ licenses/by-nc/4.0) which permits unrestricted noncommercial use, distribution, and reproduction in any medium, provided the original work is properly cited. Copyright (c) 2018 by Korean Academy of Rehabilitation Medicine 
tract (TCT). The cingulum runs down to the end of the entorhinal cortex which projects to the hippocampus [2]. Previous studies with conventional magnetic resonance imaging (MRI) have reported that lesions to the Papez circuit such as in the thalamus or hippocampus are correlated with memory impairment $[2,3]$. However, these conventional images are unable to show whether interconnected pathways of the Papez circuit are injured in the limbic system three-dimensionally. Recently, diffusion tensor tractography (DTT) has allowed for the Papez circuit to be visualized three-dimensionally and analyzed in a quantitative manner [4]. Furthermore, memory impairment in patients with brain injury has been shown to be associated with lesions on the entire neural tract in the Papez circuit three-dimensionally [5-9]. However, to date, no study has reported on the association between memory impairment and the integrity of the entire tract of the Papez circuit after multiple cerebral infarcts. Therefore, the objective of this study was to clarify the mechanism of memory impairment in a patient with concurrent thalamic and hippocampal infarctions. All four neural tracts related to the Papez circuit were reconstructed using DTT. Our findings may contribute to the elucidation of the mechanisms involved in the memory impairment in patients with cerebral infarction.

\section{CASE REPORT}

A 20-year-old male soldier abruptly experienced motor weakness 3 days before being referred to our department through the Department of Neurology. Initial diffusion brain MRI showed multiple cerebral infarcts on the thalamus, hippocampus, and fusiform areas (Fig. 1A). Upon admission, a physical examination revealed that the motor power of the left extremity was severely decreased. In a manual muscle test, the upper and lower extremities of the left extremity scored $4 / 5$. Other neurological examinations were normal. The patient could only walk with supervision. A month after the onset of weakness, he was able to return to normal life activities but still complained of memory impairment. We studied the cause of memory impairment with probabilistic DTT at 32 days after the onset. Neuropsychological evaluation was performed at 43 days after the onset. Diffusionweighted imaging data were analyzed using the Oxford Centre for Functional Magnetic Resonance Imaging of the Brain (FMRIB) Software Library. Fiber tracking was performed with the probabilistic tractography method
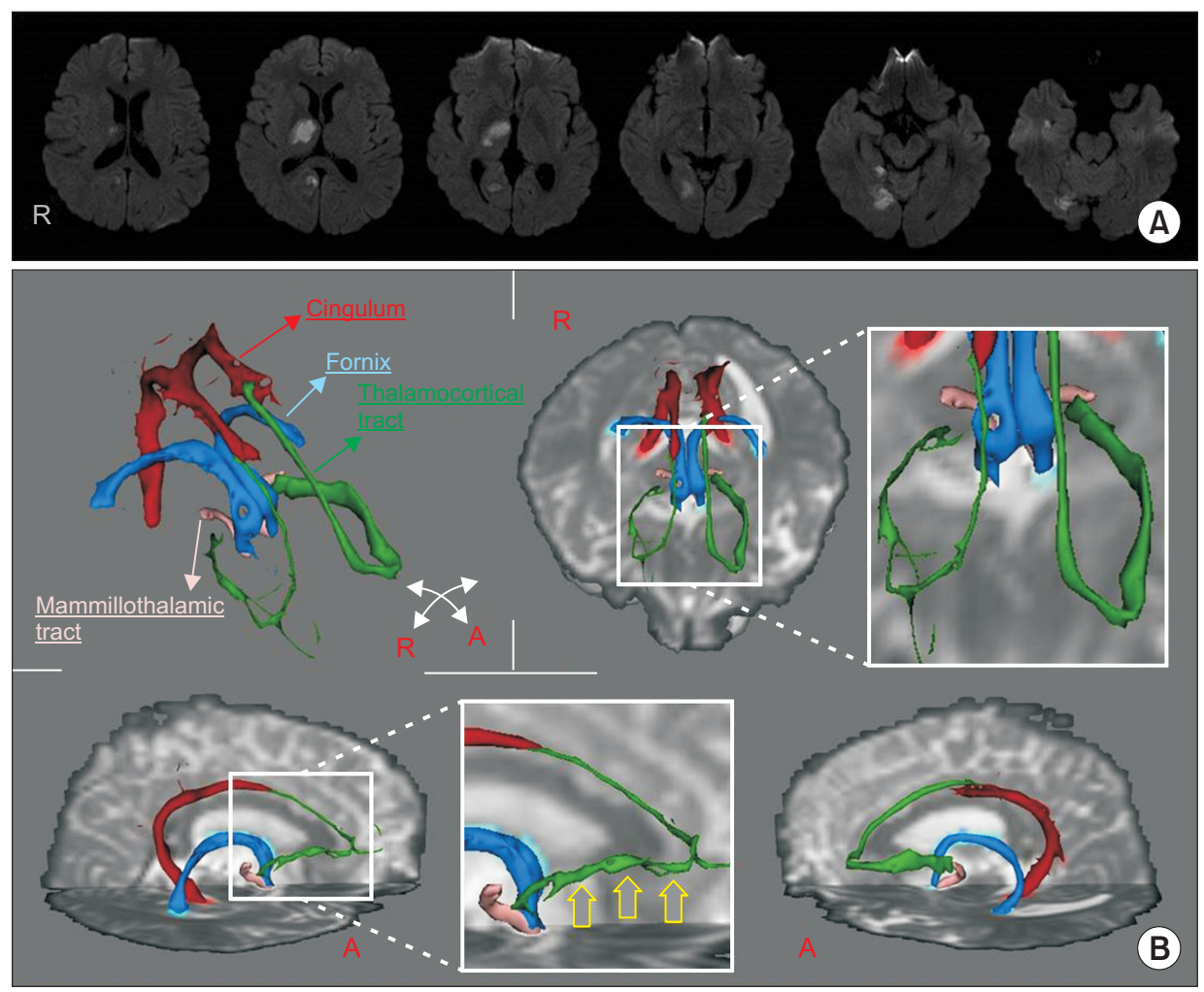

Fig. 1. (A) Diffusion brain magnetic resonance imaging showed cerebral infarction in the anterior thalamus, hippocampus, and the partially fusiform area on the right hemisphere. (B) The right thalamocortical tract (green color) between the anterior thalamic nuclei and the cingulate gyrus is thinner compared to the left thalamocortical tract (yellow arrow). Other tracts such as the mammillothalamic tract (pink), fornix (blue), and cingulum (red) are well constructed without abnormality. R, right; A, anterior. 
based on a multifiber model. Tractography routines were implemented in FMRIB (diffusion for 5,000 streamline samples at $0.5 \mathrm{~mm}$ step length and curvature threshold of 0.2 corresponding to a minimum angle of $80^{\circ}$ ). The neural tracts of the Papez circuit were reconstructed [6]. The integrity of the fornix, cingulum, and MTT was wellmaintained on both hemispheres in a three-dimensional view. Although the TCT showed symmetrical appearance between both hemispheres, the right TCT between the anterior thalamic nuclei and the cingulate gyrus was shown to be thinner compared to the left TCT (Fig. 1B). Neuropsychological scores revealed the following results. First, the verbal memory scores for short-term, delay, and recognition were in the $6.81,33.72$, and 72.24 percentiles, respectively. The visual memory scores for short-term, delay, and recognition were in the $24.83,12.51$, and 64.43 percentiles, respectively. Second, his Wechsler Adult Intelligence Scale score was 103. Third, the frontal and executive functions were normal and the scores for visuospatial and attention tests were in the 92.22 and 88.10 percentiles, respectively. These findings indicated memory impairment (mainly short-term verbal memory).

\section{DISCUSSION}

In this study, an association between injury to the TCT and memory impairment after concurrent thalamic and hippocampal infarcts was elucidated using a threedimensional view of DTT.

It has been known that both the thalamus and hippocampus are involved in memory function $[2,3]$. Using DTT, it was demonstrated that the memory impairment in this patient was specifically caused by thalamic infarction, based on the fact that the fornix from the hippocampus was intact. However, our findings were in conflict with the results of previous reports. For example, Carlesimo et al. [3] have systematically analyzed memory function in patients with thalamic stroke using neuropsychological tests and have found that memory impairment in those patients is mainly related to the MTT. Another study has demonstrated that the integrity of the MTT was affected in patients with thalamic hemorrhage, thus leading to poor cognition [10]. However, these results are limited in how they can be applied to our patient. Previous studies exploring the relationship between memory impairment and the MTT have relied on a two-dimensional view alone without investigating other neural tracts such as the TCT, fornix, and cingulum. In addition, in the latter study, memory testing was performed with a MiniMental State Examination, a screening tool. On the other hand, our results correspond to the results of previous studies regarding the relationship between the neural tract and memory function evaluated with DTT. When memory impairment occurs after brain injury, damage to the TCT is the most common cause, followed by damage to the fornix and cingulum [5-9]. To elucidate the relationship between the thalamus and memory impairment, our study demonstrates that the thalamus plays a critical role in cognitive processing.

However, this study has several limitaions. First, these observations were obtained from only one case. Therefore, caution is needed when interpreting our findings, particularly in extrapolating and generalizing them. Second, the parameters of DTT were not compared to those of a normal group. Third, we were not able to examine the gray matter of the neural tracts on the two lesions, which may be a possible cause of cognitive impairment.

Nevertheless, our results suggest that the mechanism for memory impairment following cerebral infarction is related to lesions in the Papez circuit. Our findings also demonstrate that DTT might be a useful tool for clarifying injury to the neural tract in the Papez circuit.

\section{CONFLICT OF INTEREST}

No potential conflict of interest relevant to this article was reported.

\section{ACKNOWLEDGMENTS}

This research was supported by Basic Science Research Program through the National Research Foundation of Korea funded by the Ministry of Education (No. 2013R1A1A4A01013178).

\section{REFERENCES}

1. Snaphaan L, de Leeuw FE. Poststroke memory function in nondemented patients: a systematic review on frequency and neuroimaging correlates. Stroke 2007;38:198-203.

2. Kumral E, Deveci EE, Erdogan C, Enustun C. Isolated 
hippocampal infarcts: vascular and neuropsychological findings. J Neurol Sci 2015;356:83-9.

3. Carlesimo GA, Lombardi MG, Caltagirone C. Vascular thalamic amnesia: a reappraisal. Neuropsychologia 2011;49:777-89.

4. Mori S, Aggarwal M. In vivo magnetic resonance imaging of the human limbic white matter. Front Aging Neurosci 2014;6:321.

5. Jang SH, Kwon HG. Diffuse injury of the Papez circuit by focal head trauma: a diffusion tensor tractography study. Acta Neurol Belg 2017;117:389-91.

6. Chang MC, Yeo SS, Do Lee H, Jang SH. Diffusion tensor tractography in a patient with memory impairment following encephalitis. Acta Neurol Belg 2016;116:629-31.
7. Jang SH, Yeo SS. Injury of the Papez circuit in a patient with provoked confabulation following subarachnoid hemorrhage: a diffusion tensor tractography study. Acta Neurol Belg 2016;116:655-8.

8. Yang DS, Kwon HG, Jang SH. Injury of the thalamocingulate tract in the papez circuit in patients with mild traumatic brain injury. Am J Phys Med Rehabil 2016;95:e34-8.

9. Kwon HG, Chang $\mathrm{CH}$, Jang $\mathrm{SH}$. Is thalamocortical tract injury responsible for memory impairment in a patient with putaminal hemorrhage? Neural Regen Res 2015;10:321-2.

10. Kwon HG, Lee HD, Jang SH. Injury of the mammillothalamic tract in patients with thalamic hemorrhage. Front Hum Neurosci 2014;8:259. 